\title{
Leaflet Disk Device
}

National Cancer Institute

\section{Source}

National Cancer Institute. Leaflet Disk Device. NCI Thesaurus. Code C50357.

A disk-shaped valve component of a tilting disk mechanical heart valve. 